\title{
New results on the Galactic Center Helium stars
}

\author{
Thibaut Paumard ${ }^{1}$, Jean-Pierre Maillard ${ }^{1}$, and Susan Stolovy ${ }^{2}$ \\ ${ }^{1}$ Institut d'astrophysique de Paris (CNRS), 98b Bd. Arago, 75014 Paris, France \\ ${ }^{2}$ SIRTF Science Center, CalTech, MS 220-6, Pasadena, CA 91125, USA
}

Key words infrared: stars, Galaxy: center, stars: early type, stars: Wolf-Rayet, instrumentation: spectrograph, techniques: radial velocities PACS 04A25

The cluster of helium stars around Sgr $\mathrm{A}^{\star}$ has been re-observed with the BEAR spectro-imager on CFHT, in the $2.06 \mu \mathrm{m}$ helium line, at a spectral resolution of $52 \mathrm{~km} \mathrm{~s}^{-1}$ and on a field of $\simeq 40^{\prime \prime}$. This new analysis confirms and completes a previous study at a spectral resolution of $74 \mathrm{~km} \mathrm{~s}^{-1}$ and on a smaller field of $24^{\prime \prime}$, corresponding to the central parsec (Paumard et al. 2001). Nineteen stars are confirmed as helium stars. These observations led to a clear differentiation between two groups of hot stars based on their emission linewidth, their magnitude and their positions relative to Sgr $\mathrm{A}^{\star}$. The first class of 6 members is characterized by narrow-line profiles $\left(\mathrm{FWHM} \simeq 200 \mathrm{~km} \mathrm{~s}^{-1}\right.$ ) and by their brightness. The other, fainter in $\mathrm{K}$ by an average of $2 \mathrm{mag}$, has a much broader emission component of width $\simeq 1,000 \mathrm{~km} \mathrm{~s}^{-1}$. Several of the emission lines show a P Cygni profile. From these results, we propose that the narrow-line group is formed of stars in the LBV phase, while the broad-line group is formed of stars in or near the WR phase. The division into two groups is also shown by their spatial distribution, with the narrow-line stars in a compact central cluster (IRS 16) and the other group distributed at the periphery of the central cluster of hot stars. HST-NICMOS data in $\mathrm{Pa} \alpha(1.87 \mu \mathrm{m})$ of the same field reveal a similar association. The identification of the $\mathrm{Pa} \alpha$ counterpart to the He I stars provides an additional element to characterize the two groups. Bright $\mathrm{Pa} \alpha$ emitters are found generally associated with the narrow-line class stars while the weak Pa $\alpha$ emitters are generally associated with the broad-line stars. A few particular cases are discussed. This confirms the different status of evolution of the two groups of massive, hot stars in the central cluster. As a by-product, about 20 additional candidate emission stars are detected in the central, high-resolution $19^{\prime \prime}$ field from the NICMOS data.

\section{Findings from BEAR 97 He I 2.06 $\mu$ m observation}

With the BEAR spectro-imager, an imaging FTS (Maillard 2000) the central pc of the Galaxy was observed in 1997 at a spatial resolution of $0.5^{\prime \prime}$ and spectral resolution of $74 \mathrm{~km} \mathrm{~s}^{-1}$ in the He I $2.058 \mu \mathrm{m}$ domain, covering a field of $24^{\prime \prime}$. The observation provided a homogeneous set of fully resolved line profiles. The spectro-imaging data were associated with Adaptive Optics data from CFHT in the K band (Lai et al. 1997) to check the possible confusion of sources. That particular study of the helium emission-line stars in the central parsec of the Galactic Center was published in Paumard et al. (2001). The main results can be summarized as follows:

1. 16 fully resolved P Cygni emission line profiles, cleaned of ISM emission, of purely stellar origin, were extracted.

2. they were found to divide into two distinct classes, with narrow (FWHM $\simeq 200 \mathrm{~km} \mathrm{~s}^{-1}$ ) and broad-line profiles $\left(\mathrm{FWHM} \simeq 1,000 \mathrm{~km} \mathrm{~s}^{-1}\right)$.

3. a difference in $\mathrm{K}$ of $\sim 2$ mag between the two classes was measured.

4. the spatial distribution of the two groups is different, with the narrow-line objects arranged in a central cluster, and the other class dispersed in a ring beyond a radius of $\simeq 0.3 \mathrm{pc}$ from $\mathrm{SgrA}^{\star}$.

From these findings it was concluded that the group of narrow-line stars can be considered as formed of stars in the LBV phase, and the other one of stars at the WR stage. 


\section{Observations}

New BEAR data in He I $2.06 \mu \mathrm{m}$ were obtained in June 2000 at higher spectral resolution $\left(52 \mathrm{~km} \mathrm{~s}^{-1}\right)$. The field, composed of three overlapping circular sub-fields, was wider, and the signal-to-noise ratio higher by a factor of $\simeq 1.6 . \mathrm{km} \mathrm{s}^{-1}$.

$\mathrm{Pa} \alpha$ HST/NICMOS observations were taken in 1998 of the central parsec with Camera 1 (Stolovy et al.1999) and of the central 4 pc with Cameras 2 and 3 (Scoville et al. 2003). Dithered Images were taken in filters F187N centered on the $1.87 \mu \mathrm{m} \mathrm{Pa} \alpha$ line and in F190N for the nearby continuum. By subtracting a suitably scaled F190N mosaic image from the F187N mosaic, a map of the stellar and interstellar Pa $\alpha$ emission can be obtained. Figure 3 shows the central region of the composite Camera 2 and Camera $3 \operatorname{Pa} \alpha$ image, for which the central $19^{\prime \prime} \times 19^{\prime \prime}$ has a spatial resolution of $0 .{ }^{\prime \prime} 18$.

\section{He I stars and $\mathrm{Pa} \alpha$ emission}

With the new BEAR data, almost all the stars mentioned in Paumard et al. (2001) are confirmed, except the star numbered "N6". The Pa $\alpha$ data show a bright, very small ISM feature and no stellar counterpart to this point-like He I emission. Four new broad-line stars are added. Two were out of the previously studied field, and the better signal-to-noise ratio is responsible for the other two new detections. The star "B5" was associated with IRS 13E. Maillard et al. (2003) have shown that there was indeed two emission line stars in the IRS 13E complex, namely IRS 13E2 and IRS 13E4. The broad line clearly detected in He I belongs to E2 only from Fabry-Perot imaging associated with adaptive optics by Clénet et al. (2003). The line profiles and locations of the 19 stars are shown in Fig. 1 and Fig. 2a. Fig. 2b clearly confirms that the narrow-line stars are generally much brighter in $K$ than the broad-line stars. For B11, the signal-to-noise ratio is just sufficient to claim a detection, but not to derive reliable line parameters.

Table 1 Physical properties of the helium stars: K magnitude, full width at zero intensity (FWZI) of the $2.06 \mu \mathrm{m} \mathrm{HeI} \mathrm{line}\left(\mathrm{km} \mathrm{s}^{-1}\right), \mathrm{Pa} \alpha$ line flux in units of $10^{-20} \mathrm{~W} \mathrm{~cm}^{-2}$, calibrated from $\mathrm{Pa} \alpha$ emission in $\mathrm{AF}$ (Najarro et al. 1994). ID 180 is from the photometric list of Ott et al. (1999), HeI N3 is from Paumard et al. (2001).

\begin{tabular}{|l|l|rcr|}
\hline ID & Name & $\mathrm{m}_{K}$ & $\mathrm{FWZI}_{H e I}$ & $\phi_{P a_{\alpha}}$ \\
\hline \hline N1 & IRS 16NE & 8.8 & 210 & 3.46 \\
N2 & IRS 16C & 9.6 & 490 & 2.14 \\
N3 & IRS 16SW & 9.4 & 520 & 2.09 \\
N4 & IRS 16NW & 9.9 & 470 & 1.15 \\
N5 & IRS 33SE & 9.9 & 460 & 1.12 \\
N7 & IRS 34W & 11.8 & 500 & 0.14 \\
\hline
\end{tabular}

\begin{tabular}{|l|l|rcc|}
\hline ID & Name & $\mathrm{m}_{K}$ & $\mathrm{FWZI}_{H e I}$ & $\phi_{P a_{\alpha}}$ \\
\hline \hline B1 & ID 180 & 12.4 & 1,400 & 0.40 \\
B2 & IRS 7E2 & 12.2 & 1,500 & 0.28 \\
B3 & IRS 9W & 11.8 & 1,900 & 0.83 \\
B4 & IRS 15SW & 11.5 & 1,400 & 0.76 \\
B5 & IRS 13E2 & 11.0 & 1,300 & 2.27 \\
B6 & IRS 7W & 12.0 & 1,600 & 0.44 \\
B7 & AF & 10.6 & 1,200 & 4.40 \\
B8 & AFNW & 11.5 & 1,800 & 1.05 \\
B9 & HeIN3 & 12.7 & 1,600 & 0.14 \\
B10 & BSD WC9 & 10.6 & 2,000 & 0.37 \\
B11 & IRS 29N & 9.9 & 1,100 & 0.07 \\
B12 & IRS 15NE & 11.2 & 1,500 & 0.37 \\
B13 & IRS 16SE2 & 11.8 & 940 & 1.15 \\
\hline
\end{tabular}

Fig. 3a shows that the He I stars are associated with the Pa $\alpha$ emission stars. The narrow-line stars (circles) are coincident with bright $\mathrm{Pa} \alpha$ emitters (mean intensity $\simeq 210^{-20} \mathrm{~W} \mathrm{~cm}^{-2}$ without taking into account IRS 34W, Table 1), whereas the broad-line stars correspond to fainter Pa $\alpha$ emitters (mean intensity $\simeq 0.9710^{-20} \mathrm{~W} \mathrm{~cm}^{-2}$ ). Other $\mathrm{Pa} \alpha$ emitters are present, which may be also associated with He I emission, but too faint to have been detected with BEAR. A source extraction with the StarFinder procedure (Diolaiti et al. 2000) gives 52 point-like emission features in the high-resolution 19" central field, of which 43 are emission line stars with a high degree of certainty. The 9 other need further observation to rule out the possibility that these point sources are compact ISM features or incomplete continuum subtraction of stars. However, this result (Fig. 3b) is generally consistent with an independent analysis made by Scoville et al. (2003). 


\section{Discussion}

The central cluster of helium stars is confirmed with a total of 19 members currently identified. The various conclusions on the characteristics of these stars from the first paper, reviewed in the Introduction are confirmed: division into two groups from their linewidths (Fig. 1 and Fig. 2b), from their brightness and from their location (Fig. 2a). The difference of brightness (Fig. 2b) presents few exceptions which were already noticed in the first paper. One of the narrow-line stars (N7, IRS 34W) is weak and one of the broad-line stars (B11, IRS 29N) is brighter than the average of the other stars of the same group. From a long-term photometric study (Ott et al. 1999) IRS 34W is indicated as a variable star. It was weak at the time of our observations (Ott et al., private communication).

It was proposed in Paumard et al. (2001) that the group of bright, helium stars was made of LBV-type stars. The high intensity of $\mathrm{Pa} \alpha$ (Table 1), the variability of IRS $34 \mathrm{~W}$ confirm that these stars are hot, mass-losing stars, still rich in hydrogen. On the contrary, the weakness of the Pa $\alpha$ emission combined with the very broad helium line are consistent with the other group being more evolved stars. A few sources are exceptions - IRS 13E2, AF, IRS 16SE2 - showing a broad-line He I profile, but strong Pa $\alpha$ emission. This apparent anomaly could certainly be due to the fact that the $\mathrm{Pa} \alpha$ filter is not perfectly adapted to distinguish between rich and poor hydrogen emitters. Since the $\mathrm{Pa} \alpha$ line is blended with another significative helium line, $\mathrm{He} \mathrm{I}(4-3)$ at $1.869 \mu \mathrm{m}$, the intensity detected by the F187N can remain strong even if the hydrogen emission is intrinsically weak. Already mentioned, the weakness of the K magnitude of IRS 34W (Table 1) is due to the star being in a phase of enhanced intrinsic extinction. Naturally, the measured $\mathrm{Pa} \alpha$ intensity is extremely weak, a factor 14 lower than the mean intensity. However, all these elements confirm the different status of evolution of the two groups of massive, hot stars in the central cluster. Assuming that all these stars were formed in the same star formation event, the differences in evolutionary state would come from the differences in their initial mass.

The $\mathrm{Pa} \alpha$ data can help to address the question of whether the identification of emission line stars in the central region is complete or not. Possibly, about twenty new stars, associated with weak Pa $\alpha$ emission are detected in the central parsec (Fig. 3b). With only this indication, it cannot be concluded that they are more WR candidates. A deep, spectroscopic analysis using adaptive optics in the $\mathrm{K}$ band is needed. Besides more WRs, some of them could be Be stars, or could belong to the old star population as symbiotic or Mira-type stars in a phase of emission. At any rate, these data represent a new element in the census of spectral type in the central parsecs to better constrain the peculiar star formation conditions in this region of the Milky Way.

\section{References}

Clénet, Y., et al.2003, these proceedings

Diolaiti E., Bendinelli, O., Bonaccini, D., Close, L., Currie, D., \& Parmeggiani, G. 2000, A\&AS, 147, 335

Lai, O., et al. 1997, In: Optical Telescopes of Today and Tomorrow, A.L. Ardeberg (ed), Proc. SPIE 2871,859

Maillard, J.P., 2000, In: Imaging the Universe in 3 Dimensions, E. van Breughel \& J. Bland-Hawthorn (eds), ASP Conf. Serie 195, 185

Maillard, J.P., Paumard, T., Stolovy, S.R., \& Rigaut, F. 2003, these proceedings

Najarro, F., Hillier, D.J., Kudritzki, R.P., Krabbe, A., Genzel, R., Lutz, D., Drapatz, S., \& Geballe, T.R. 1994, A\&A, 285, 573

Ott, T., Eckart, A., \& Genzel, R. 1999, ApJ, 523, 248

Paumard, T., Maillard, J.P., Morris, M., \& Rigaut, F. 2001, A\&A, 366, 466

Scoville, N.Z., Stolovy, S.R., Rieke, M., Christopher, M., \& Yusef-Zadeh, F. 2003, ApJ, submitted

Stolovy, S.R., McCarthy, D.W., Melia, F., Rieke, G., Rieke, M.J., \& Yusef-Zadeh, F., 1999, in The Central Parsecs of the Galaxy, ed. H. Falcke, A. Cotera, W.J. Duschl, F. Melia, M.J. Rieke, ASP Conf. Ser., 186, 39 


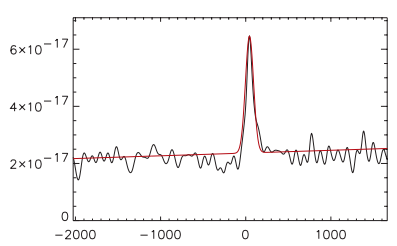

$\mathrm{N}$ 1)
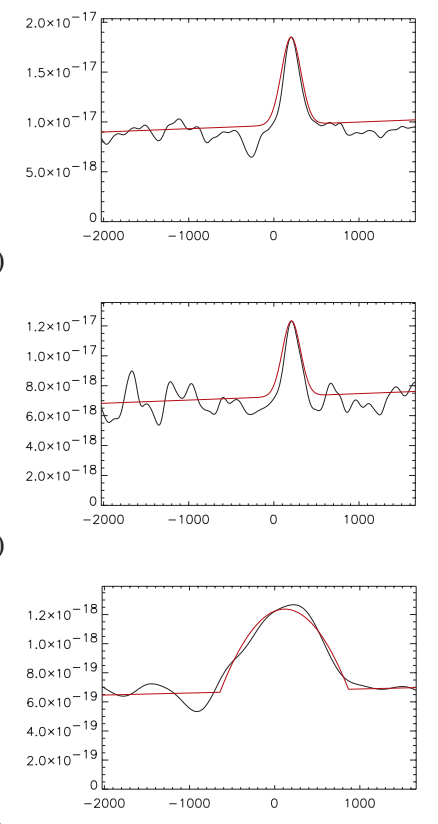

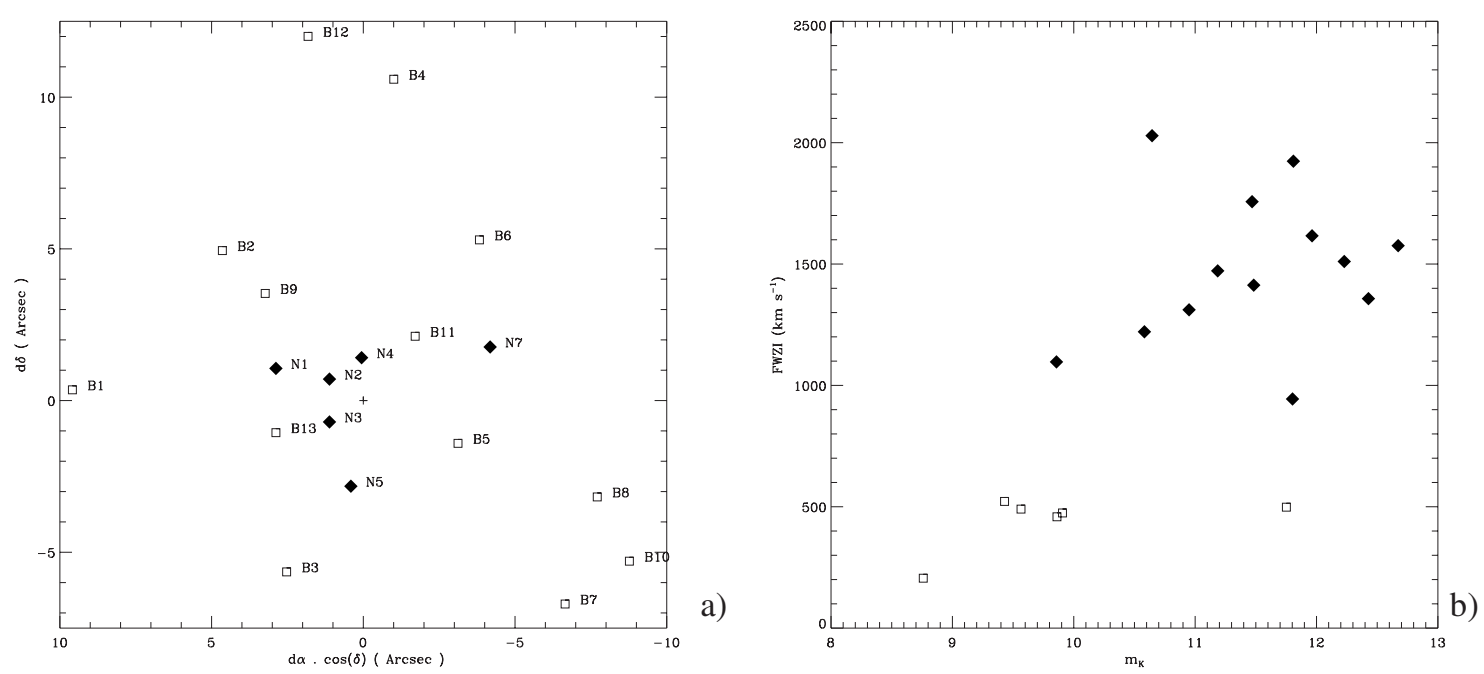

Fig. 2 a) Positioning of the helium stars, b) full width at zero intensity (FWZI) against K magnitude plot. The empty squares represent the broad-line stars, while the filled diamonds represent the narrow-line stars. On plot b), two regions can be seen: all the narrow-line stars, except IRS $34 \mathrm{~W}$ - that is variable (see text) -, are grouped with a $K$ magnitude brighter than 10, and all the broad-line stars, except one, have a $K$ magnitude fainter thant 10.5 (mean value $\simeq 12$ ).
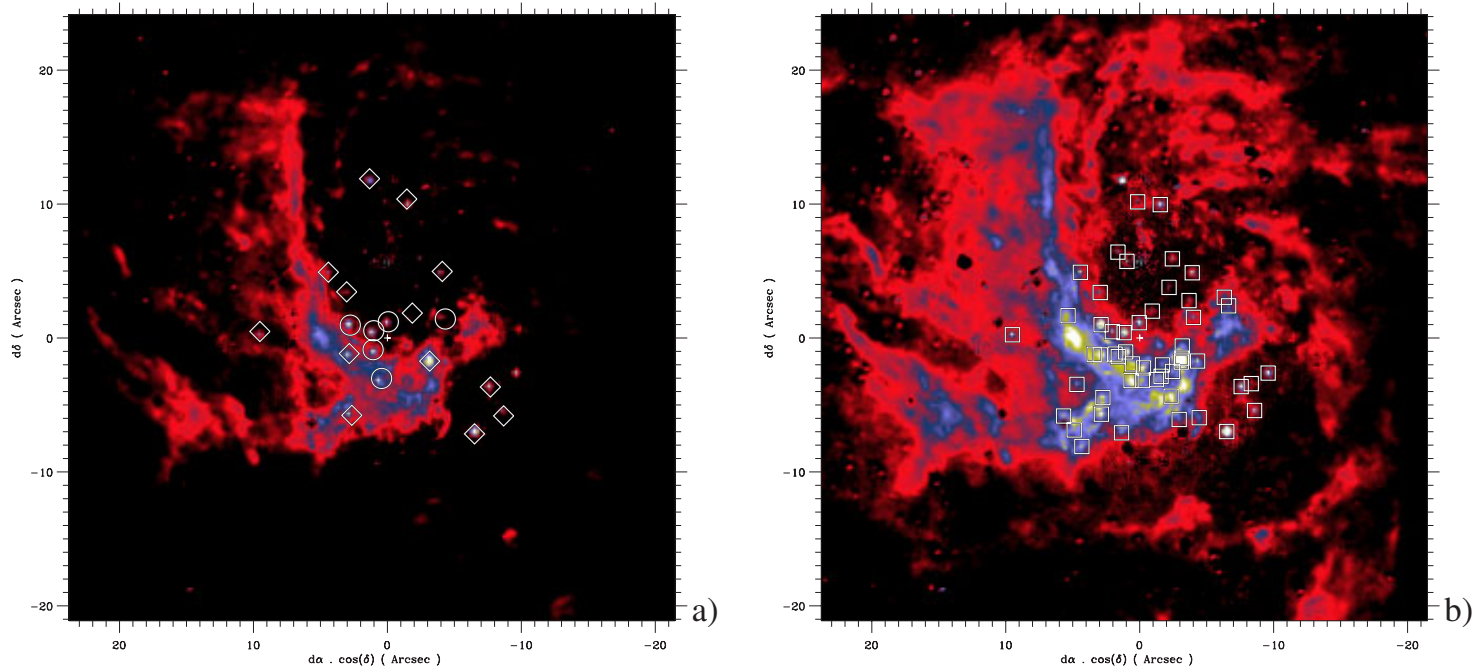

Fig. 3 a) Pa $\alpha$ map of the central 2 pc, with the inner $19^{\prime \prime}$ comprised of the high resolution Camera 2 data. Diamonds indicate the locations of the broad-line stars while the narrow-line stars are indicated by circles. All of these stars show emission in this $\mathrm{Pa} \alpha$ filter. b) All emission line star candidates, in the central region, marked by square boxes. The intensity scale is stretched to show fainter emission. 